\title{
Expression of tissue inhibitor of metalloproteinases-1 and B-cell lymphoma-2 in the synovial membrane in patients with knee osteoarthritis
}

\author{
XIAOMIAO LI, XIAODONG FU, YINGJIAN GAO, HAO LI, WEILI WANG and YI SHEN
}

Department of Orthopedics, Ren Ji Hospital, School of Medicine, Shanghai Jiao Tong University, Shanghai 200127, P.R. China

Received June 1, 2017; Accepted September 25, 2017

DOI: $10.3892 / \mathrm{etm} .2017 .5462$

\begin{abstract}
The purpose of this study was to determine the expression and impact of tissue inhibitor of metalloproteinases-1 (TIMP-1) and B-cell lymphoma-2 (Bcl-2) in knee osteoarthritis (KOA). We collected synovial fluids from the knee joint of $70 \mathrm{KOA}$ patients and 30 controls. The expression levels of TIMP-1 and Bcl-2 were significantly higher in KOA patients than those in the control group $(\mathrm{P}<0.01)$. We also found positive correlation between the severity of KOA and the expression level of TIMP-1 ( $\mathrm{r}=0.8027, \mathrm{P}<0.05)$ and and $\mathrm{Bcl}-2$ $(\mathrm{r}=0.5336, \mathrm{P}<0.05)$. However, we found no correlation between the expression levels of TIMP-1 and Bcl-2 in the synovial membranes of KOA patients ( $\mathrm{P}>0.05)$. Both TIMP-1 and $\mathrm{Bcl}-2$ are expressed at high levels in the synovial membrane with $\mathrm{KOA}$, and are closely related to the occurrence and development of KOA. Thus, detection of TIMP-1 and Bcl-2 in KOA patients can be helpful in diagnosing the state of KOA.
\end{abstract}

\section{Introduction}

Osteoarthritis is a degenerative disease affecting the joints caused by multiple factors. Osteoarthritis is mainly featured by progressive articular cartilage injury, subchondral bone sclerosis, and formation of osteophyte around the joints; arthralgia (pain in the joint) is the most typical clinical manifestation (1-3). In clinical practice, osteoarthritis is treated mainly by relieving the symptoms and improving joint function with the main objective of improving quality of life. In most cases, patients with end-stage knee osteoarthritis can only get effective treatment through joint replacement surgery, but the surgery has important risks, including large trauma, high costs and high failure rates. Therefore, it is crucial to find effective methods for early diagnosis of osteoarthritis that can

Correspondence to: Dr Yi Shen, Department of Orthopedics, Ren Ji Hospital, School of Medicine, Shanghai Jiao Tong University, 160 Pujian Road, Shanghai 200127, P.R. China

E-mail: yi_shenrj@hotmail.com

Key words: knee osteoarthritis, B-cell lymphoma-2, tissue inhibitor of metalloproteinases-1, synovial fluid prevent further degeneration that lead to surgery (4-6). Knee osteoarthritis (KOA) is a common form of osteoarthritis that is the most extensively studied at present. Much attention has been paid to the role of tissue inhibitor of metalloproteinases (TIMP) in KOA in recent years. TIMP-1 is a new member of the TIMP family widely distributed that inhibits specific matrix metalloproteases (MMPs) wiht a crucial role in the occurrence and development of KOA (7). B-cell lymphoma/ leukemia-2 (Bcl-2) is an anti-apoptotic protein that promotes cell survival by inhibiting apoptosis. Studies report that the expression level of Bcl-2 may influence the occurrence and development of arthritis (8).

Here, we explored the relationship between the expression levels of TIMP-1 and Bcl-2 in synovial tissues and the clinicopathologic characteristics of KOA, hoping to provide new insights for the early diagnosis of KOA.

\section{Subjects and methods}

Research subjects. We enrolled 70 patients who came for medical consultation, received treatment, and were diagnosed with KOA by experts in Renji Hospital from August, 2014 to August, 2015. Patients were aged 45-87 years, including 32 men and 30 women. Patients were not affected by other degenerative conditions. We completed clinical and pathological data for all patients as well as treatment protocols. The control group consisted of 30 healthy people who received physical examination, in which arthritis and other degenerative diseases were excluded. The diagnosis of KOA was done according to the KOA diagnostic criteria recommended by the American College of Rheumatology in 2000 (9). Severity classification of KOA was performed according to Lawrence radiographic grading criteria (10): mild (joint space $\geq 7 \mathrm{~mm}$, with formation of osteophyte in the joint space), moderate (joint space at 4-7 mm, with obvious osteophyte formed), and severe (joint space disappeared, with massive osteophyte). All patients signed informed consent before enrolling in the study. The study was approved by the Ethics Committee of Ren Ji Hospital, School of Medicine, Shanghai Jiao Tong University.

Collection and preservation of synovial fluid. For collection of synovial fluid in knee joint, the patient was placed in the supine position with the knee extended. The puncture point was located at the junction of the lateral edge of the quadriceps 
A

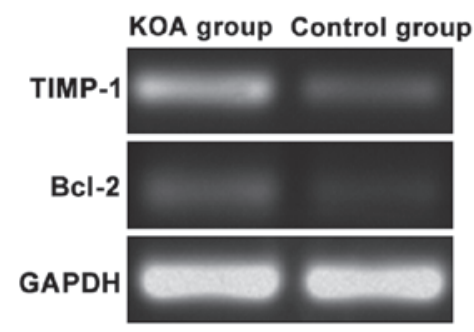

B

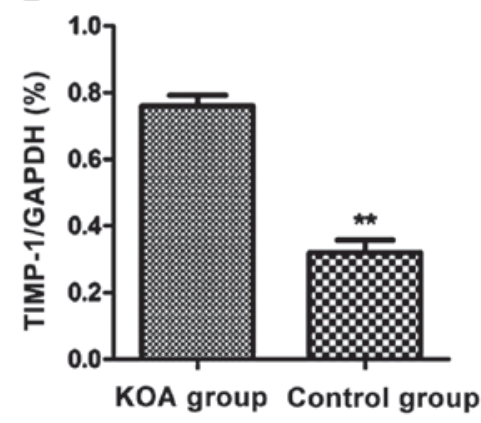

C

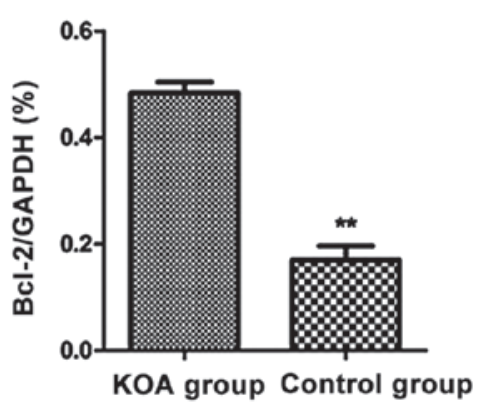

Figure 1. Expression of tissue inhibitor of metalloproteinases-1 (TIMP-1) and B-cell lymphoma-2 (Bcl-2) mRNA in synovial membranes of knee osteoarthritis (KOA) patients and controls via semi-quantitative RT-PCR. (A) Agarose gel electrophoresis. (B and C) Statistical charts for the relative expression of TIMP-1 and Bcl-2. TIMP-1 and Bcl-2 are significantly elevated in the synovial tissues of KOA patients compared to the control group ("** $<<0.01)$.

Table I. Sequence of PCR primers.

\begin{tabular}{ll}
\hline Gene & \multicolumn{2}{c}{ Primer sequences } \\
\hline TIMP-1 & F: 5'-ATCCACCTTGACGATGCTTTAC-3' \\
& R: 5'-TTCAGATGTTCTAAGCCTACGG-3' \\
Bcl-2 & F: 5'-TGGCCCTCGTAGCCTTGAGGAC-3' \\
& R: 5'-CCAGTGCTGCAGGGTCCGAGGT-3' \\
GAPDH & F: 5'-GATGATTGGCATGGCTTT-3' \\
& R: 5'-CACCTTCCGTTCCAGTTT-3'
\end{tabular}

TIMP-1, tissue inhibitor of metalloproteinases-1; Bcl-2, B-cell lymphoma-2; GAPDH, glyceraldehyde 3-phosphate dehydrogenase; $\mathrm{F}$, forward; R, reverse.

femoris muscle and the line joining the superior edge of the patella. Synovial fluid (0.5-2 ml) was withdrawn from the knee joint and collected in a tube with heparin. The synovial fluid was snap-frozen after it was centrifuged within $2 \mathrm{~h}$ and then it was placed at $-80^{\circ} \mathrm{C}$ for long-term storage.

Semi-quantitative reverse transcriptase PCR (RT-PCR). Total RNA in the synovial fluids was extracted according to the instructions of the TRIzol kit (Invitrogen, Carlsbad, CA, USA). Agarose gel electrophoresis was used to confirm RNA integrity. The $28 \mathrm{~S}, 18 \mathrm{~S}$ and $5 \mathrm{~S}$ bands were clear and the $28 \mathrm{~S}$ band was twice as bright as the $18 \mathrm{~S}$ band, indicating that the RNA could be used in subsequent experiments. The RNA was reversely transcribed into cDNA with the reverse transcription kit (Invitrogen). The expression levels of TIMP-1 and $B c l-2$ mRNA were detected by semi-quantitative PCR (Thermo Fisher Scientific GmbH, Dreieich, Germany) from synovial fluids of KOA patients and controls, using glyceraldehyde 3 -phosphate dehydrogenase $(G A P D H)$ as internal control. Reaction conditions: 35 cycles of $30 \mathrm{sec}$ at $95^{\circ} \mathrm{C}, 25 \mathrm{sec}$ at $64^{\circ} \mathrm{C}$ and $30 \mathrm{sec}$ at $72^{\circ} \mathrm{C}$. Primers were synthesized by Tiangen Biotech (Beijing, China) (Table I). When the reaction was completed, they were observed under the ultraviolet imaging system (Biometra GmbH, Göttingen, Germany) after agarose gel electrophoresis, and the relative expression of TIMP-1 and $B c l-2$ were presented normalized to $G A P D H$.
Enzyme-linked immunosorbent assay (ELISA). Hydrochloric acid (HCL) $(0.1 \mathrm{mmol})$ was added to synovial fluids at 1:5 (w:v). The synovial fluids were sonicated with ultrasonic waves and then centrifuged at $1,000 \mathrm{xg}$ for $15 \mathrm{~min}$ at $4^{\circ} \mathrm{C}$ (ABI, Carlsbad, CA, USA). The precipitate was discarded and the supernatant was collected. The $\mathrm{pH}$ of the fluid was adjusted to 7.0 using $1 \mathrm{mmol}$ sodium hydroxide. The expression levels of TIMP-1 and Bcl-2 proteins were measured according to the instructions of ELISA kit (R\&D Systems, Minneapolis, MN, USA), the absorbance of the samples was measured with a microplate reader (Thermo Fisher Scientific $\mathrm{GmbH}$ ), and the contents of TIMP-1 and Bcl-2 in each group of samples were calculated.

Statistical analysis. Data were presented as mean \pm standard deviation. SPSS 19.0 software was used for data processing and analysis (SPSS, Chicago, IL, USA). t-test was used for measurement data and $\chi^{2}$ test was used to analyze enumeration data groups. Homogeneity of variance test was performed; if there was homogeneity of variance, the Bonferroni method was used for pairwise comparison. If there was heterogeneity of variance, the Welch method was be used for analysis. Dunnett's T3 method was used for multiple comparisons and Pearson's correlation analysis was used for correlation analysis. $\mathrm{P}<0.05$ suggested that the difference was statistically significant.

\section{Results}

Expression of TIMP-1 and Bcl-2 mRNA in synovial membranes in KOA. We used the synovial fluids from KOA patients and normal controls to analyze the expression level of TIMP-1 and $B c l-2$ mRNA via semi-quantitative RT-PCR. The relative expression of TIMP-1 and $B c l-2$ were significantly increased in the synovial fluids of KOA patients compared with those in the control group $(\mathrm{P}<0.01)$ (Fig. 1).

Expression of TIMP-1 and Bcl-2 protein in synovial membranes in KOA. We next used the synovial fluids from KOA patients and normal controls to analyze the expression level of TIMP-1 and Bcl-2 proteins by ELISA. The relative expression of TIMP-1 and Bcl-2 were significantly increased in the synovial fluids of KOA patients compared with those in the control group $(\mathrm{P}<0.01)$ (Fig. 2). 
A

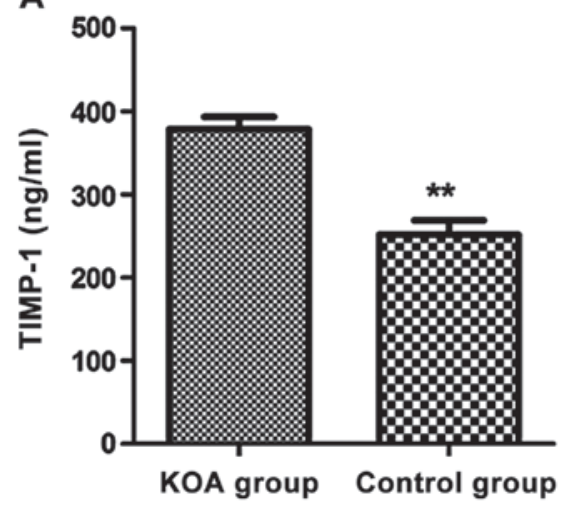

B

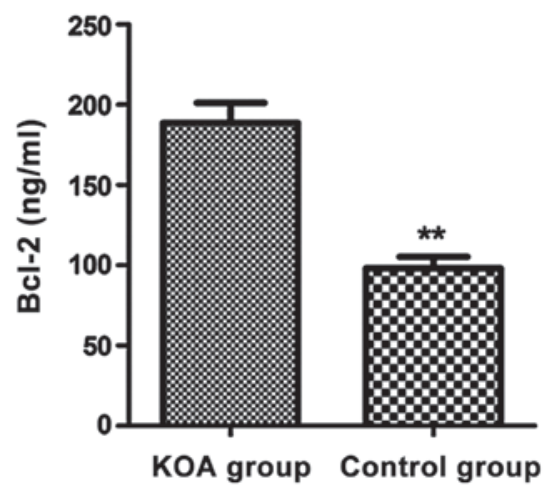

Figure 2. Relative expression of tissue inhibitor of metalloproteinases-1 (TIMP-1) and B-cell lymphoma-2 (Bcl-2) in synovial membranes of knee osteoarthritis (KOA) patients and normal controls via enzyme-linked immunosorbent assay (ELISA). (A) Expression level of TIMP-1. (B) Expression level of Bcl-2. The relative expression of TIMP-1 and Bcl-2 are significantly higher in the synovial tissues of KOA patients than those in the control group $\left({ }^{* *} \mathrm{P}<0.01\right)$.

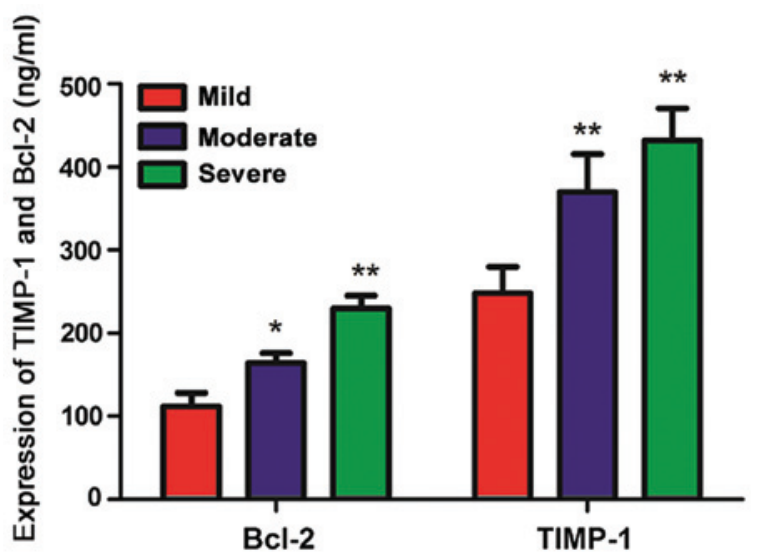

Figure 3. Expression of tissue inhibitor of metalloproteinases-1 (TIMP-1) and B-cell lymphoma-2 (Bcl-2) in synovial membranes of patients with varying severity of knee osteoarthritis (KOA). TIMP-1 and Bcl-2 are significantly higher in the synovial tissues in moderate and severe KOA than those in mild KOA (vs. mild KOA ${ }^{*} \mathrm{P}<0.05$ and ${ }^{* *} \mathrm{P}<0.01$ ).

Expressions of TIMP-1 and Bcl-2 and clinico-pathology. Next, we analyzed the expression of TIMP-1 and Bcl-2 in the synovial tissues of KOA patients in combination with age, sex, and the severity and relapse of KOA. The relative expression of TIMP-1 and Bcl-2 were not correlated with the age and sex of patients (Table II). However, they were closely related to the severity and relapse of KOA (Table II). The relationship between the severity of KOA and the expression levels of TIMP-1 and Bcl-2 is shown in Fig. 3.

Correlation between expression levels of TIMP-1 and Bcl-2 and KOA. We next performed Pearson's correlation analysis to analyze the relationship between the expression levels of TIMP-1 and Bcl-2 and the severity of KOA. The expression level of TIMP-1 was positively correlated with the severity of KOA ( $r=0.8027, \mathrm{P}<0.05)$ (Fig. 4). The expression level of Bcl-2 was also positively correlated with the severity of KOA $(\mathrm{r}=0.5336, \mathrm{P}<0.05)$ (Fig. 5).

Statistical analysis was performed on the expression levels of TIMP-1 and Bcl-2 in the synovial tissues of KOA patients. When the expression level was higher in KAO patients than that of controls, it was considered as high expression. If

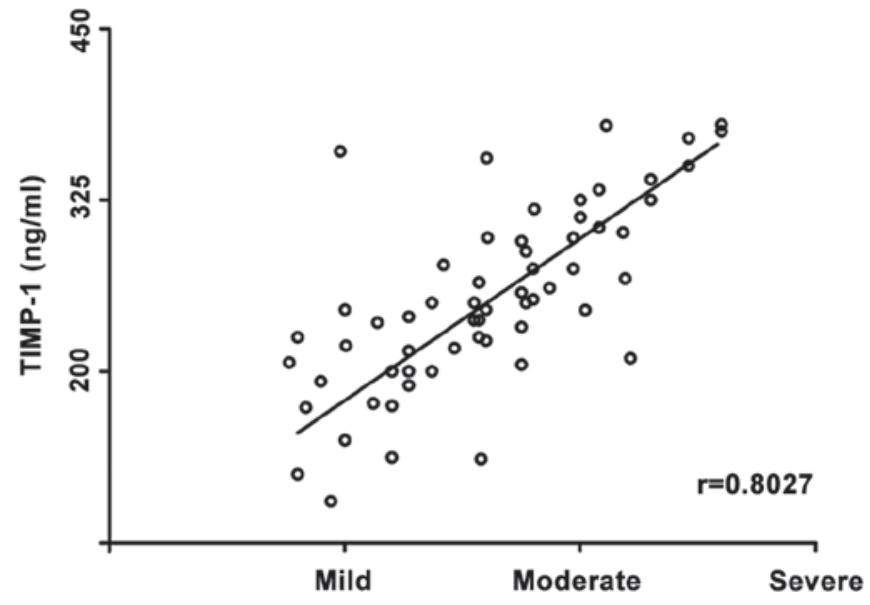

Figure 4. Correlation between expression level of tissue inhibitor of metalloproteinases-1 (TIMP-1) and severity of knee osteoarthritis (KOA). The expression level of TIMP-1 is positively correlated with the severity of KOA.

expression in $\mathrm{KAO}$ patients was equal to or lower than that of controls, it was considered normal or low expression. The expression levels of TIMP-1 and Bcl-2 in the synovial tissues of KOA patients were not mutually correlated $(\mathrm{r}=-0.658$, $\mathrm{P}>0.05$ ) (Table III).

\section{Discussion}

The onset of KOA is a dynamic, progressive process in which subchondral bone and articular cartilage influence each other. The pathogenesis of KOA often leads to changes in stress stimulation of subchondral bone on articular cartilage and, eventually, causes destruction of articular cartilage, inducing the onset of osteoarthritis (11). Currently the diagnosis for KOA is fairly delayed and patients cannot be treated in a timely manner. Furthermore, with the progressive aging of the population, the KOA patients and the society as a whole are facing huge economic pressures. With the continuous progress of molecular biology and genetic engineering, scholars in China and other countries have made in-depth analyses on osteoarthritis from the perspective of pathogenesis, providing new approaches for the treatment and diagnosis of $\operatorname{KOA}(12,13)$. 
Table II. Relationship between relative expression of MMP-2 and TIMP-3 and clinical characteristics.

\begin{tabular}{lcccc}
\hline Category & $\mathrm{n}$ & Expression of TIMP-1 $(\mathrm{ng} / \mathrm{ml})$ & P-value & Expression of Bcl-2 (ng/ml) \\
\hline $\begin{array}{l}\text { Age (years) } \\
\leq 60\end{array}$ & 34 & $373.58 \pm 56.33$ & 0.0692 & $185.53 \pm 21.08$ \\
$>60$ & 36 & $364.16 \pm 49.28$ & $176.85 \pm 19.12$ \\
Sex & & & 0.0782 \\
Male & 32 & $367.52 \pm 53.08$ & $164.63 \pm 33.62$ \\
Female & 38 & $353.96 \pm 61.22$ & $172.57 \pm 25.29$ \\
Severity of KOA & & & 0.012 & 0.0873 \\
Mild & 13 & $248.26 \pm 31.39$ & $112.08 \pm 16.19$ \\
Moderate & 32 & $369.89 \pm 45.87$ & & $163.95 \pm 12.01$ \\
Severe & 35 & $431.86 \pm 38.65$ & & $229.72 \pm 15.32$ \\
Relapse & & $375.68 \pm 29.86$ & 0.021 \\
Yes & 28 & $304.52 \pm 25.36$ & & $201.87 \pm 11.37$ \\
No & 42 & & & $115.96 \pm 16.69$ \\
\hline
\end{tabular}

MMP, matrix metalloproteinase; TIMP, tissue inhibitor of metalloproteinases; KOA, knee osteoarthritis.

Table III. Correlation between expressions of MMP-2 and TIMP-3 in KOA.

TIMP-1

\begin{tabular}{|c|c|c|c|c|}
\hline Bcl-2 & High expression & Normal or low expression & r-value & P-value \\
\hline High expression & 12 & 58 & -0.658 & 0.081 \\
\hline Normal expression or low expression & 49 & 21 & & \\
\hline
\end{tabular}

MMP, matrix metalloproteinase; TIMP, tissue inhibitor of metalloproteinases; KOA, knee osteoarthritis.

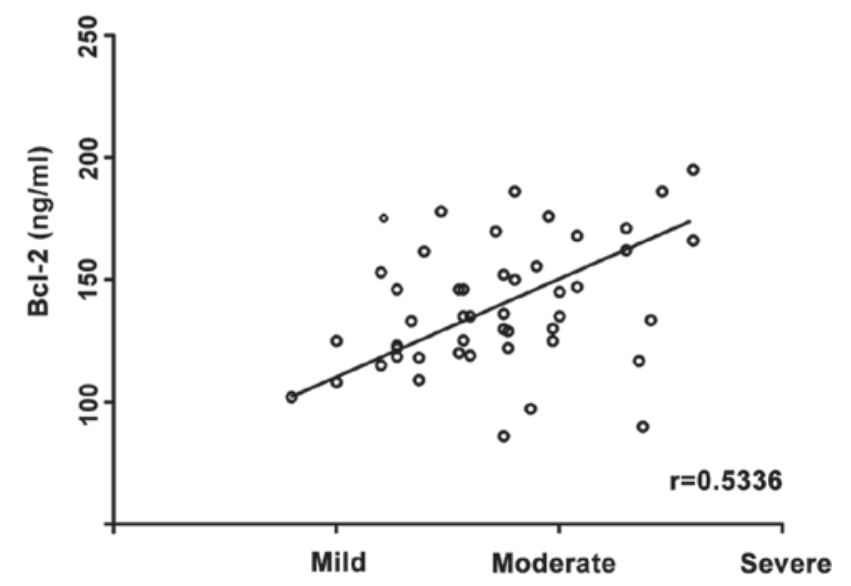

Figure 5. Correlation between expression level of B-cell lymphoma-2 (Bcl-2) and severity of knee osteoarthritis (KOA). The expression level of Bcl-2 is positively correlated with the severity of KOA.

MMPs are calcium- and zinc-dependent proteases that can degrade almost all the components of extracellular matrix. Studies published in the past few years indicate that MMPs are related to onset of KOA (14). For instance, MMPs and TIMP plays a crucial role in the pathogenesis of KOA (15). TIMP can hydrolyze the glycoproteins in the articular cartilage, destroy the cartilaginous skeleton, and accelerate the progression of KOA. TIMP-1 plays an important role in the pathogenesis of several inflammatory conditions (16). Here, we found that the levels of TIMP-1 mRNA and protein were significantly increased in the synovial tissues of KOA patients compared with those in controls. We also found that the expression level of TIMP-1 in patients with severe KOA was significantly higher than that in patients with milder KOA. Our analysis showed positive correlation between the expression level of TIMP-1 and the severity of osteoarthritis. Our results indicate that TIMP-1 is closely related to the onset and development of KOA. Batra et al found that TIMP regulated the metabolic processes of the extracellular matrix by inhibiting MMPs (7). When TIMP was expressed at high levels, the TIMP-1/MMPs ratio aggravated cartilage injury and affected the development of osteoarthritis. In a normal cartilage, there is a balance between MMPs and MMPs inhibitors: the imbalance between MMPs and MMPs inhibitors may lead to cartilage degeneration (17). Therefore, early diagnosis of KOA can be realized by detecting the expression level of TIMP-1 in the synovial membrane of KOA patient.

Bcl-2 is known to be the most important anti-apoptotic cellular factor. Bcl-2 is mainly distributed in the serosal surface of outer mitochondrial membrane, nuclear membrane, and endoplasmic reticulum surface. Bcl-2 stabilizes the 
mitochondrial membranes and maintains transmembrane potentials in the mitochondria (17). Some studies also find that Bcl-2 can reduce chondrocyte apoptosis by maintaining the integrity of the extracellular matrix and regulating the activity of telomerase (18). A study found that the expression level of Bcl-2 adjacent to the fissure of cartilage was higher than that in cartilage without a fissure and higher expression level was detected in areas with severe injury (19). Here, we showed that the expression levels of $\mathrm{Bcl}-2$ were significantly higher in synovial tissues of KOA patients than those in controls. The expression level of Bcl-2 was closely related to the severity of arthritis, but there was no correlation between the expression levels of Bcl-2 and TIMP-1 in KOA patients. These results suggest that $\mathrm{Bcl}-2$ participates in the occurrence and development of KOA, and that $\mathrm{Bcl}-2$ may be involved in the regulation of articular chondrocyte apoptosis in KOA patients. The pathogenesis of KOA may be closely related to chondrocyte apoptosis and the expression level of Bcl-2 can be used as one of the indicators for diagnosing KOA.

In conclusion, both TIMP-1 and Bcl-2 are closely related to the occurrence and development of KOA. Thus, they can be used as the indicators for clinical diagnosis of KOA. However, there are still some limitations to our study. The sample size is small and all the patients were enrolled in one hospital. We did not perform mechanistic studies to determine the causative role of TIMP-1 and Bcl-2 on the pathogenesis of KOA. Nevertheless, TIMP-1 and Bcl-2 may be valuable for clinical diagnosis of KOA, and TIMP-1 and Bcl-2 can bring about new breakthroughs for clinical diagnosis and treatment of KOA.

\section{References}

1. Kuyinu EL, Narayanan G, Nair LS and Laurencin CT: Animal models of osteoarthritis: Classification, update, and measurement of outcomes. J Orthop Surg 11: 19, 2016.

2. Bruyère $\mathrm{O}$, Cooper $\mathrm{C}$, Arden $\mathrm{N}$, Branco $\mathrm{J}$, Brandi ML, Herrero-Beaumont G, Berenbaum F, Dennison E, Devogelaer JP, Hochberg M, et al: Can we identify patients with high risk of osteoarthritis progression who will respond to treatment? A focus on epidemiology and phenotype of osteoarthritis. Drugs Aging 32: 179-187, 2015.

3. Arden N, Richette $\mathrm{P}$, Cooper $\mathrm{C}$, Bruyère $\mathrm{O}$, Abadie $\mathrm{E}$, Branco $\mathrm{J}$, Brandi ML, Berenbaum F, Clerc C, Dennison E, et al: Can we identify patients with high risk of osteoarthritis progression who will respond to treatment? A focus on biomarkers and frailty. Drugs Aging 32: 525-535, 2015.

4. Moreau M, Pelletier JP, Lussier BV, d'Anjou MA, Blond L, Pelletier JM, del Castillo JR and Troncy E: A posteriori comparison of natural and surgical destabilization models of canine osteoarthritis. BioMed Res Int 33: 851-855, 2013.

5. Heijink A, Gomoll AH, Madry H, Drobnič M, Filardo G, Espregueira-Mendes J and Van Dijk CN: Biomechanical considerations in the pathogenesis of osteoarthritis of the knee. Knee Surg Sports Traumatol Arthrose 20: 423-435, 2012.

6. Musumeci G, Castrogiovanni P, Trovato FM, Weinberg AM, Al-Wasiyah MK, Alqahtani MH and Mobasheri A: Biomarkers of chondrocyte apoptosis and autophagy in osteoarthritis. Int J Mol Sci 16: 20560-20575, 2015.
7. Batra J, Robinson J, Mehner C, Hockla A, Miller E, Radisky DC and Radisky ES: PEGylation extends circulation half-life while preserving in vitro and in vivo activity of tissue inhibitor of metalloproteinases-1 (TIMP-1). PLoS One 7: e50028, 2012.

8. Foight GW and Keating AE: Locating herpesvirus Bcl-2 homologs in the specificity landscape of anti-apoptotic Bcl-2 proteins. J Mol Biol 427: 2468-2490, 2015.

9. Rother S, Samsonov SA, Moeller S, Schnabelrauch M, Rademann J, Blaszkiewicz J, Köhling S, Waltenberger J, Pisabarro MT, Scharnweber D, et al: Sulfate hyaluronan alters endothelial cell activation in vitro by controlling the biological activity of the angiogenic factors vascular endothelial growth factor-A and tissue inhibitor of metalloproteinase-3. ACS Appl Mater Interfaces 9: 9539-9550, 2017.

10. McConnell S, Kolopack P and Davis AM: The Western Ontario and McMaster Universities Osteoarthritis Index (WOMAC): A review of its utility and measurement properties. Arthritis Rheum 45: 453-461, 2001.

11. Valverde-Franco G, Lussier B, Hum D, Wu J, Hamadjida A, Dancause N,Fahmi H,Kapoor M,Pelletier JP and Martel-Pelletier J: Cartilage-specific deletion of ephrin-B2 in mice results in early developmental defects and an osteoarthritis-like phenotype during aging in vivo. Arthritis Res Ther 18: 65, 2016.

12. Lourenço S, Lucas R, Araújo F, Bogas M, Santos RA and Barros H: Osteoarthritis medical labelling and health-related quality of life in the general population. Health Qual Life Outcomes 12: 146, 2014.

13. Kim C, Nevitt MC, Niu J, Clancy MM, Lane NE, Link TM, Vlad S, Tolstykh I, Jungmann PM, Felson DT, et al: Association of hip pain with radiographic evidence of hip osteoarthritis: Diagnostic test study. BMJ 351: h5983, 2015.

14. Adamson A, Ghoreschi K, Rittler M, Chen Q, Sun HW, Vahedi G, Kanno Y, Stetler-Stevenson WG, O'Shea JJ and Laurence A: Tissue inhibitor of metalloproteinase 1 is preferentially expressed in Th1 and Th17 T-helper cell subsets and is a direct STAT target gene. PLoS One 8: e59367, 2013.

15. Tency I, Verstraelen H, Kroes I, Holtappels G, Verhasselt B, Vaneechoutte M, Verhelst R and Temmerman M: Imbalances between matrix metalloproteinases (MMPs) and tissue inhibitor of metalloproteinases (TIMPs) in maternal serum during preterm labor. PLoS One 7: e49042, 2012.

16. Hire JM, Evanson JL, Johnson PC, Zumbrun SD, Guyton MK, McPherson JC III and Bojescul JA: Variance of matrix metalloproteinase (MMP) and tissue inhibitor of metalloproteinase (TIMP) concentrations in activated, concentrated platelets from healthy male donors. J Orthop Surg 9: 29, 2014.

17. Dhar A, Gardner J, Borgmann K, Wu L and Ghorpade A: Novel role of TGF- $\beta$ in differential astrocyte-TIMP-1 regulation: Implications for HIV-1-dementia and neuroinflammation. J Neurosci Res 83: 1271-1280, 2006.

18. Lee SY, Kwok SK, Son HJ, Ryu JG, Kim EK, Oh HJ, Cho ML, Ju JH, Park SH and Kim HY: IL-17-mediated Bcl-2 expression regulates survival of fibroblast-like synoviocytes in rheumatoid arthritis through STAT3 activation. Arthritis Res Ther 15: R31, 2013.

19. Kernt M, Neubauer AS, Eibl KH, Wolf A, Ulbig MW, Kampik A and Hirneiss C: Minocycline is cytoprotective in human trabecular meshwork cells and optic nerve head astrocytes by increasing expression of XIAP, survivin, and Bcl-2. Clin Ophthalmol 4: 591-604, 2010.

This work is licensed under a Creative Commons Attribution-NonCommercial-NoDerivatives 4.0 International (CC BY-NC-ND 4.0) License. 